\title{
La tecnología en el aprendizaje del estudiantado de la Facultad de Ciencias Químicas, Universidad Central del Ecuador
}

Technology in the learning of university students of the Faculty of Chemical Sciences, Central University of Ecuador

\section{Volumen 20, Número 2 \\ Mayo - Agosto \\ pp. 1-37}

\author{
Elithsine E. Espinel Armas
}

\section{Citar este documento según modelo APA}

Espinel Arman, Elithsine E. (2020). La tecnología en el aprendizaje del estudiantado de la Facultad de Ciencias Químicas, Universidad Central del Ecuador. Revista Actualidades Investigativas en Educación, 20(2), 1-37. Doi. 10.15517/aie.v20i2.41653 


\title{
La tecnología en el aprendizaje del estudiantado de la Facultad de Ciencias Químicas, Universidad Central del Ecuador
}

Technology in the learning of university students of the Faculty of Chemical Sciences, Central University of Ecuador

\author{
Elithsine E. Espinel Armas ${ }^{1}$
}

\begin{abstract}
Resumen: Las condiciones actuales, generadas por el desarrollo tecnológico, tienen gran influencia en los modos de actuación y comportamiento de los seres humanos. En esta realidad se incluye el sistema de educación, por ello el presente artículo buscó determinar el criterio del sector estudiantil universitario sobre la utilización y aporte de la tecnología en el proceso de enseñanza-aprendizaje (PEA). Como consecuencia, la investigación se apoyó en el enfoque cuantitativo, el nivel alcanzado es descriptivo con apoyo de investigación transversal de campo y bibliográfica. La población objeto de estudio estuvo conformada por 122 estudiantes con matrícula en el segundo semestre durante el período marzo-agosto 2019. El instrumento de recolección de datos fue sometido a validación y cálculo de confiabilidad estadística, previa su aplicación. La información recolectada fue procesada mediante estadística descriptiva. Los resultados relevantes señalan que el sector estudiantil estima el uso de las nuevas tecnologías en su aprendizaje, por lo que las TIC de acceso a la información, conectividad y expositivas son elementos que requieren ser incorporados de manera pedagógica a dicho proceso como elementos mediadores del aprendizaje, que al ser planificados y aprovechados eficientemente en el aula, permiten el alcance de habilidades y capacidades, así como la generación de ambientes de trabajo que promueven interacción positiva entre docentes y estudiantes en un clima de reflexión y autonomía.
\end{abstract}

Palabras clave: Proceso de enseñanza, universidad, habilidades, TIC.

Abstract: The big technological development that the currently society is facing up has huge implications in many different fields, one of them, on the education system. That's why, this research study aimed to identify the opinion that university student sector have regarding the use and contribution of technology in the teaching-learning process. This descriptive study used a quantitative approach with support of cross-field research and documentary. The population included: 122 students enrolled in the second semester during the period 20192019. The instrument for data collection was subjected to validation and calculation of statistical reliability, prior to its application. The information collected was processed by descriptive statistics. This study showed that students estimate the use of new technologies in their learning. Also, the new technologies like searching tools, digital access of information, connectivity and slides/presentation software are elements that must be pedagogically incorporated in the teaching learning process. These technologies will allow the students to reach the maximum capacities and abilities in a setting with a good student-teacher relationship. At the same time, giving the students the autonomy and self-reflection they need for the development of their own learning.

Key words: Teaching process, university, skills, ICT.

\footnotetext{
${ }_{1}$ Docente Titular de la Universidad Central del Ecuador, ECUADOR, Nivel Agregado, de Grado y Posgrado de la Facultad de Ciencias Químicas. ORCID: https://orcid.org/00000001-5800-7035?lang=es
}

Dirección electrónica: elyespinel@yahoo.es, eeespinel@uce.edu.ec

Artículo recibido: 18 de octubre, 2019

Enviado a corrección: 5 de febrero, 2020

Aprobado: 20 de abril, 2020 


\section{Introducción}

En la época actual, se evidencia una verdadera revolución en el ámbito científico y tecnológico, lo cual manifiesta sustanciales cambios en lo económico, psicosocial, cultural, comportamental y, por ende, en el ámbito educacional. Así, la tecnología, como recurso de aula, se convierte en una herramienta imprescindible que posibilita la apropiación de conocimientos y el fortalecimiento de habilidades en el empleo de los medios de información y comunicación.

La incorporación de las Tecnologías de Información y Comunicación (TIC) en la gestión de aula deberán apoyar la ejecución de las actividades de aprendizaje, las cuales, según el Reglamento de Régimen Académico, deben organizarse en los siguientes componentes: “ a) Aprendizaje en contacto con la persona docente b) Aprendizaje autónomo y c) Aprendizaje práctico-experimental..." (Consejo de Educación Superior-CES-, 2019, p.13 ); pero además, las TIC generan cambios en la práctica del sector docente, en lo referente al desarrollo micro curricular, sin son utilizadas pertinentemente en el alcance de los objetivos educativos, consolidados en el perfil de egreso. (CES, 2013). Frente a esta realidad surge el cuestionamiento: ¿El uso y aporte de las TIC en el Proceso de Enseñanza Aprendizaje (PEA) permite el desarrollo de habilidades en el estudiantado de la Facultad de Ciencias Químicas?

Bajo este antecedente, es interesante resaltar las cifras publicadas por el Instituto Ecuatoriano de Estadísticas y Censos (INEC), el cual señala que, a nivel nacional, en 2017, el 40,7\% utilizó internet como fuente para obtener información; 31\% como medio de comunicación; 21,1\% para educación-aprendizaje; y 3,3\% con fines de trabajo (INEC, 2017). Asimismo, en Ecuador, en el 2018, el acceso de los hogares a internet tiene una participación del 37,2\%, es decir, un incremento del 14,7\% en relación con el año 2012. "De igual manera, en el área urbana y rural se evidencia un crecimiento significativo de 15,2 y 11,3, respectivamente" (INEC, 2018, p. 6), lo que evidencia la mejor accesibilidad de la población en porcentajes porcentuales.

En Ecuador en el año 2018, sus habitantes, realizaron las actividades señaladas anteriormente, mediante conexión a internet en su hogar el 66,2\%, en su lugar de trabajo el $11,6 \%$ en su institución educativa el 7,2\%, en centros de acceso público el 10,2\% y el 3,9\% en casa de otra persona (INEC, 2018). 
Sobre el uso del internet para el aprendizaje en el Ecuador, en la Tabla 1, se muestra un análisis comparativo que indica la reducción de su utilización con fines académicos, lo que contrasta pese a la mayor posibilidad de acceso a medios de conexión digital.

Tabla 1

Ecuador: Porcentaje de participación en el uso de internet con fines de educación y aprendizaje por año

\begin{tabular}{|cc|}
\hline AÑO & PARTICIPACIONN \\
\hline 2010 & $40,0 \%$ \\
\hline 2011 & $29,1 \%$ \\
\hline 2012 & $27,5 \%$ \\
\hline 2013 & $31,7 \%$ \\
\hline 2014 & $26,5 \%$ \\
\hline 2015 & $25,4 \%$ \\
\hline 2016 & $23,2 \%$ \\
\hline 2017 & $21,1 \%$ \\
\hline
\end{tabular}

Fuente: Elaboración propia a partir de la información del INEC $(2016,2018)$

Otro elemento a considerar se refiere a la calidad y a la fiabilidad de la información en el ambiente digital, pues el libre acceso a publicaciones y la carencia de filtros de control previos, provocan que los contenidos de sus trabajos, informes bibliográficos, análisis de casos, entre otros, no posean la relevancia requerida en la formación universitaria. Es evidente la poca utilización de fuentes digitales confiables, como Google Académico, Microsoft Academic Search, Dialnet, YouTube Educación, Education Resources Information Center, Redalyc, RefSeek, y de manera específica, en la Universidad Central del Ecuador, pese a contar con un importante repositorio digital y bases de datos como Digitalia, Micromedex, Scopus, Springer Link, entre otros, su recurrencia por parte del estudiantado aún es limitada. Situación que desemboca en reticencia por parte de quienes ejercen la docencia al momento de efectuar tareas académicas que requieren revisión bibliográfica, pues se tiene la percepción sobre la limitada capacidad estudiantil en seleccionar fuentes confiables de información y por ende, el riesgo de trabajos de baja calidad.

En este sentido, Gallent y Tello ( 2017) sostienen:

Ciertamente las nuevas formas de reproducir y distribuir la información han fomentado la creencia de que todo vale, todo puede descargarse y todo puede utilizarse en beneficio propio dado que es de acceso público, induciendo así al uso ilegítimo de obras de terceros (p. 92) 
Hay que destacar que, la facilidad de acceso a la red por el bajo costo, así como las habilidades del alumnado en el manejo de tecnología pueden limitar su desempeño académico a únicamente buscar, copiar y pegar textos e imágenes, desaprovechando estos recursos en la generación de aprendizajes significativos y provocadores de competencias (Climént, 2018). Por ello, se hace necesario promover en el estudiantado el respeto a la creación o autoría de su semejante, así como evaluar la calidad y confiabilidad de la información que se despliega en los medios tecnológicos.

En este contexto de globalidad, en el Ecuador las TIC provocan un nuevo reto al sistema educativo, el cual debe transitar de un modelo unidireccional, tradicional y lineal de formación, centrado en la transmisión de los saberes desde las personas expertas o sus sustitutos, los libros de texto, hacia formas más flexibles de interactuación radial. Esta nueva realidad exige mayor flexibilidad, con preponderancia hacia el desarrollo de propuestas creativas e innovadoras, a fin de estimular acciones colaborativas. De esta manera, la información situada en grandes bases de datos pueda ser compartida entre el estudiantado en nuevos entornos o contextos no tangibles sino virtuales, posibilitando la interactuación académica. (Gavilanes, Yanza, Inca, Torrez, y Sánchez, 2019). Entonces el aula se modifica, de un conjunto arquitectónico y cultural estable a un ciberespacio dúctil y amplio. Ese es el nuevo escenario de la educación, ante el cual docentes y alumnado deben adoptar estrategias que contribuyan a la apropiación efectiva del conocimiento.

Sin embargo, pese a que el uso educativo de las herramientas tecnológicas tiene mayor cobertura y alcance, la limitación está asociada a la facilidad para acceder, tanto a las herramientas de acceso a la información como de conectividad, siendo responsabilidad del estado la dotación de recursos para reducir la brecha para acceder a la tecnología y alcanzar resultados educativos de calidad. Al respecto, Díaz (2014), citado por Hermosa (2015) señala:

La posibilidad de acceso pasará a ser un factor más de desigualdad en el que se encuentran muchos jóvenes. En consecuencia, la sofisticación tecnológica responde a que la educación no debe contenerse con aceptar un futuro previsible, se debe permitir la participación activa de los estudiantes en las herramientas educativas y contenidos temáticos que les motivan (p.126)

Es imperante una transformación o, en un más amplio sentido, una revolución educativa, a fin de eliminar las brechas que se manifiestan entre quienes pueden pagar una 
educación privada frente a quienes tienen que aspirar y esperar por un cupo universitario, lo que refleja un síntoma prevalente de exclusión y desigualdad. Así también, este cambio implica la asignación de recursos desde el Estado para las mejoras de infraestructura y tecnologías, aunque esta es una realidad difícil de superar, en virtud de que la asignación presupuestaria cada vez es más reducida, y de esta más del $90 \%$ se destina para gastos corrientes, y el restante casi $10 \%$ se distribuye entre gasto de inversión, de capital y de financiamiento. (Secretaría de Educación Superior, Ciencia, Tecnología e Innovación 2019)

En lo concerniente al tema abordado, las actuales demandas sociales y económicas han contribuido a que, el sector profesional graduado de tercer nivel, busque como una alternativa continuar sus estudios de posgrado en modalidades: on line a distancia o semipresencial. En este sentido, una significativa experiencia en uso de TIC constituye la capacitación que brindan universidades nacionales e internacionales o centros de educación continua, a través de los Cursos Abiertos Masivos On Line (MOOCS) o formación universitaria de grado como de la Universidad Particular de Loja o Universidad Internacional de La Rioja, en Ecuador. En el caso de la Universidad Central, la modalidad a distancia actualmente se centra en la formación de grado en carreras de Educación, mientras que en los programas de posgrado existe una importante oferta bajo la modalidad semipresencial. Por ello, merece especial atención el fortalecimiento de habilidades que impliquen mayor interacción y manejo de las TIC, lo cual apoyará a que su adaptación y participación en estas modalidades de estudio sean más efectivas.

De esta manera, el presente artículo tiene como objetivo: analizar el uso de las TIC y su aporte en el PEA para el desarrollo de habilidades en estudiantes de segundo semestre, período marzo-agosto 2019 de la Facultad de Ciencias Químicas, de la Universidad Central del Ecuador.

\section{Breve referente teórico}

La incorporación de las TIC en el contexto formativo implica un reto para el sistema educativo, así como importantes transformaciones en el PEA, en su planificación, organización y evaluación, la reestructuración de los modelos educativos, de los escenarios o contextos del aprendizaje y la asunción de nuevos roles y habilidades de los actores del proceso. Siendo una realidad que, el estudiantado que se incorpora al ámbito universitario, dispone de habilidades digitales y comunicacionales, en contraste con las competencias digitales limitadas en los docentes (Durán, Barragán, González, y Guzmán, 2017). 
Lo que se busca destacar es que, la integración de las nuevas tecnologías en el PEA, a más de ser concebidas como estrategias educativas, recursos didácticos, objetos de estudio, factores clave del proceso investigativo o elementos generadores de la comunicación y de difusión del pensamiento, son componentes importantes de la gestión tanto académica como administrativa.

Así pues, la nueva sociedad basada en el manejo de la información genera nuevas relaciones entre docentes, autoridades, estudiantes, familias y el entorno mismo. De manera que el uso de la tecnología: "No es una simple adaptación, sino un proceso creativo en el que recíprocamente las herramientas facilitan las prácticas y las prácticas innovadoras se crean con el fin de hacer un mejor uso de las nuevas posibilidades que ofrecen las tecnologías" (Gros, 2016, p.3). Entonces, no se trata de utilizar la tecnología, por parte del profesorado, solamente como medio de transferencia y disposición de información, sino la adopción de verdaderos cambios en la práctica académica; por ello, es necesario que propicien rupturas de las viejas tradiciones pedagógicas a través del aprovechamiento del ciberespacio y sus potencialidades, así como de las habilidades del estudiantado, "nativos digitales", al tener un mayor acercamiento al manejo de redes, gestión en la búsqueda de información, facilidad de manejo de dispositivos digitales.

Sobre el efecto de dicha incorporación de las TIC en el ámbito educacional, Area (2008), citado por Durán et al.(2017), plantea:

(...) el proceso de cambio se puede ver reflejado cuando un profesor decide emplear las nuevas tecnologías en su docencia, pues indica que se están planteando nuevos retos y desafíos, lo que implica conocimiento, habilidades, cambio de actitudes y tiempo; dicho comportamiento e inversión de tiempo no es un proceso espontáneo y azaroso, sino que tiene que ver con un modelo educativo, el cual involucra los procesos de enseñanza-aprendizaje, la institución, los estudiantes y los docentes. (p.84)

Los nuevos roles que desempeñan en el aula, tanto docentes como estudiantes, conlleva adoptar y adaptar las TIC al proceso educativo, a fin de generar ambientes que procuren el mejoramiento de los modelos y paradigmas de enseñanza en acción. De manera que se logre el empoderamiento mediante el aporte de las experticias particulares de los participantes del PEA (González, 2017), a fin de que se provoque un espacio reflexivo y colaborativo que promueva una formación integral e integradora. Sobre lo tratado, se señala: 
(...) las diferencias positivas que conlleva el uso de la tecnología se encuentran relacionadas con las estrategias de aprendizaje empleadas. Es decir, las condiciones en que se produce un aprendizaje poseen un rol fundamental en la incorporación de las TIC en el aula. Por tanto, las creencias del profesor en torno a las características del proceso de aprendizaje se convierten en un elemento fundamental para el uso de las TIC. (Tapia, 2018, p.11)

Otro elemento favorable de los medios tecnológicos, es favorecer a los sectores vulnerables, ya que su accesibilidad facilita la democratización de recursos bibliográficos, la mayor participación en comunidades de aprendizaje, rompe las barreras geográficas y trasciende los muros del salón de clases para una formación más cooperativa.

Ahora bien, en el afán de estar acordes con los requerimientos de modernización, las instituciones realizan inversiones importantes para adquirir equipos, software, medios audiovisuales, entre otros. Sin embargo, los resultados no son los esperados, ya que usar tecnología para lograr habilidades y capacidades va mucho más allá, solo la integración planificada y facilitada por docentes permitirá un uso pertinente con la labor educativa para el desarrollo de aprendizajes significativos. Así, y dadas las condiciones económicas de las instituciones, sobre todo públicas, hay graves dificultades para el equipamiento de medios tecnológicos, lo que provoca acrecentamiento de la brecha digital, la cual es una lamentable realidad en Latinoamérica. En concordancia, se plantea:

La brecha digital se refiere a la diferenciación o separación producida entre individuos, grupos de individuos, instituciones, sociedades o países en el acceso y uso de las TIC. Puede ser definida en términos de la desigualdad de posibilidades que existen para acceder a la información, el conocimiento y la educación a través de la tecnología. (Sánchez, Reyes, Ortiz y Olarte, 2018, p.116)

Las herramientas tecnológicas que el medio ofrece a docentes y estudiantes se clasifican en:

- herramientas para acceso a la información: laptop, IPad, Smartphone, teléfonos inteligentes;

- herramientas de conectividad y coordinación de tareas: bluetooth, internet, intranet, Wi-Fi;

- herramientas expositivas: Prezi, Power Point, Youtube; 
- plataformas tecnológicas: Moodle; buscadores: google académico, google libros, entre otros.

Aunque hay diversidad de herramientas, son susceptibles de ser mal utilizadas, por parte del alumnado durante el proceso educativo como un elemento distractor, y por docentes al no incorporarlas de manera planificada en el aula. Este elemento es vital, pues si las y los docentes incorporan herramientas tecnológicas se disponen a una nueva organización curricular, con estrategias que den una dinámica nueva al PEA, caso contrario, su utilización no garantiza una intención formativa, sino meramente instrumental. Al ser el modelo pedagógico constructivista, brújula de la tarea docente centralina, en su acción debe permitir al estudiantado reflexionar sobre los procedimientos pertinentes para llegar a la apropiación del conocimiento y alcance de habilidades.

Al respecto, Echeverría (2015) señala en su estudio:

Esta incorporación de las TIC en el currículo educativo presenta limitaciones, las cuales se originan por diversos factores. El manejo por parte del profesorado de las herramientas tecnológicas a veces es pobre o inexistente, así como su conocimiento de las posibles aplicaciones didácticas. (p. 4)

En lo referente a la evaluación, la literatura ofrece diversos softwares especializados, los cuales, para su correcta aplicación, requerirán la formación o capacitación previa tanto de docentes como de estudiantes, a fin de superar las deficiencias de los softwares que se basan solamente en evaluar contenidos mediante aplicación de pruebas objetivas de respuesta cerrada. Entre los más destacados se establecen:

1. Entornos virtuales de formación (web-based trining), que ayudan al profesorado a gestionar un módulo o curso de enseñanza completo a través de la red (distribución de contenidos, intercambios con los alumnos a través de correo electrónico, foros de discusión 0 chats y evaluación de los alumnos). Ejemplos de este tipo: WebCT, Learning Space, Edustance, etc.

2. Herramientas de autor, consisten en un software destinado, a su vez, a la creación de programas a modo de ejercicios o tareas. Ejemplos de este tipo son: Hot Potatoes, Quia!, Clic, etc.

3. Software específico más complejo (requiere manejo de servidor), que permite poner en la red a toda la institución, tanto para la creación y publicación de los exámenes, como 
para recoger los resultados de los estudiantes. Ejemplos de este software son: Perception y Quiz Factory (sic). (Rodríguez, 2005, parr. 33)

\section{Metodología}

\subsection{Enfoque}

Para cumplir el propósito, en el presente estudio se realizó el análisis de la información otorgada por el estudiantado del segundo semestre de la Facultad de Ciencias Químicas de la Universidad Central del Ecuador sobre la tecnología en su PEA. Por tanto, se sustentó en el paradigma de investigación cuantitativo, pues los resultados obtenidos fueron tabulados y analizados estadísticamente (Baptista, Collado, y Hernández, 2014). En tanto que el nivel investigativo alcanzado es descriptivo, pues se evidencia el uso y aporte de la tecnología en el aprendizaje del sector estudiantil universitario para el desarrollo de habilidades. La investigación es de tipo transversal de campo, así, se realizó la recolección de información en un solo corte y en el escenario universitario, lugar donde se evidencia el problema.

\subsection{Unidades de análisis}

La población, entendida como un "conjunto de personas, hechos, elementos, entre otros, sobre los cuales se desea estudiar un fenómeno o acontecimiento" (Espinel, 2015, p. 51), se estableció con estudiantes universitarios de la Facultad de Ciencias Químicas, como informantes pertinentes para emitir juicio respecto del uso y aporte de la tecnología en su PEA.

El criterio de inclusión consideró: estudiantes con matrícula legalizada para el segundo semestre, correspondiente al período académico marzo-agosto 2019 , de las dos carreras vigentes por rediseño: de Bioquímica y Farmacia y de Química.

El criterio de exclusión establecido correspondía a: personas sin matrícula legalizada para el segundo semestre de las carreras vigentes, así como a estudiantes con o sin matrícula legalizada de las carreras cerradas habilitadas para titulación: Química de alimentos, Química Farmacéutica, Bioquímica Clínica y Química.

En la cuantificación de las personas que cumplían el criterio de inclusión, se determinó que correspondían a 126 individuos, al ser una población de fácil acceso para la investigadora, se decidió trabajar con la totalidad.

Al momento de la aplicación del instrumento de recolección de datos, acudieron 122 discentes ( $97 \%$ del total de la población), a quienes se les informó sobre el objetivo del 
estudio y se manifestó el compromiso de confidencialidad con la información suministrada. Por ello, se solicitó su consentimiento de participación mediante la explicación previa.

Tabla 2

Población estudiantil por carrera, Facultad de Ciencias Químicas, Universidad Central del Ecuador, 2019

\begin{tabular}{|lc|}
\hline Carrera & Número de estudiantes \\
\hline Bioquímica y farmacia & 104 \\
\hline Química & 18 \\
\hline Población & 122 \\
\hline
\end{tabular}

Fuente: Elaboración propia de la autora (2019)

\subsection{Técnicas de recolección}

Las variables de estudio consideradas son: Uso de tecnología y Aprendizaje universitario, estas fueron entendidas desde la fundamentación epistémica y operativizadas mediante indicadores, a fin de medirlas, con apoyo de instrumentos documentales.

En la Tabla 3 se presenta la ruta operativa de las variables del estudio:

\begin{tabular}{|c|c|c|}
\hline \multicolumn{3}{|c|}{$\begin{array}{c}\text { Tabla } 3 \\
\text { Número de ítems en correspondencia con } \\
\text { variables y dimensiones del estudio }\end{array}$} \\
\hline Variables & Dimensiones & № de ítems \\
\hline \multirow[t]{3}{*}{ Uso de tecnología } & Métodos innovadores de EA & 8 \\
\hline & Incorporación TIC en aula & 1 \\
\hline & Infraestructura tecnológica & 1 \\
\hline \multirow{13}{*}{$\begin{array}{l}\text { Aprendizaje } \\
\text { Universitario }\end{array}$} & Análisis, procesamiento y redacción & 1 \\
\hline & Alcance de objetivos & 1 \\
\hline & Aprendizaje colaborativo & 1 \\
\hline & Trabajo individual & 2 \\
\hline & Trabajo autónomo & 1 \\
\hline & Logro de habilidades comunicativas & 2 \\
\hline & Habilidades investigativas & 1 \\
\hline & Habilidades operativas & 1 \\
\hline & Autonomía en aprendizaje & 1 \\
\hline & Habilidades interpersonales & 1 \\
\hline & Uso de dispositivo móvil en PEA & 1 \\
\hline & Accesibilidad a internet & 1 \\
\hline & Total & 24 \\
\hline
\end{tabular}

Fuente: Elaboración propia de la autora (2019)

Una vez operacionalizadas las variables, para la recolección de información, se optó por la técnica de la encuesta y como instrumento un cuestionario digital (Anexo 1). El cuestionario estuvo estructurado con 24 preguntas cerradas tipo Likert, con cuatro opciones 
de respuesta. Previa la aplicación del instrumento, se sometió a validación mediante juicio de tres personas versadas en el ámbito investigativo, para valorar la calidad de constructo de dicho documento, por tanto, se observó la correspondencia de cada ítem con objetivos, variables y dimensiones, así como calidad técnica, representatividad, concordancia y uso adecuado del lenguaje (Espinel, 2015). Las observaciones y recomendaciones emitidas permitieron corregir el cuestionario y estructurar su versión definitiva.

Posteriormente, se procedió a establecer la confiabilidad estadística mediante pilotaje al $10 \%$ de la muestra, es decir 13 individuos, y el cálculo del respectivo coeficiente de Alfa de Cronbach, cuyo resultado fue de 0.87 , lo que indica que el instrumento de recolección de datos tiene una alta consistencia interna y fiabilidad.

\subsection{Procesamiento de análisis}

El cuestionario estructurado se configuró a partir de las 15 dimensiones derivadas de las variables. Dicho cuestionario se aplicó de manera digital a la población de estudio. Se continuó con el proceso de análisis de resultados, para lo cual se codificaron las respuestas, se asignó otorgando 1 a la opción Nunca, 2 a Ocasionalmente, 3 a Casi Siempre, y 4 a Siempre. Se procedió a la tabulación mediante Microsoft Excel 2010 y a la construcción de tablas de frecuencias por cada ítem, y en forma global del instrumento.

El procesamiento de datos se llevó a cabo con apoyo de estadística descriptiva, ya que se estableció la frecuencia absoluta y relativa de cada opción de respuesta de los 24 ítems. Los resultados fueron expresados en porcentajes, según los indicadores establecidos.

El estudio se centró en el análisis del uso y aporte que otorgan las TIC al sector estudiantil universitario participante, distribuido en las 24 preguntas, por lo que la interpretación se realizó de cada una de ellas, para proceder con la respectiva discusión analítica con base en la interpretación de los resultados obtenidos de las y los 122 informantes.

\section{Resultados y análisis}

En la Tabla 4 se presentan 22 de los 24 ítems que tienen la misma opción múltiple, los cuales se ordena de mayor a menor según sus puntuaciones promedio. Se destaca, por parte de las personas encuestadas, el criterio mayor sobre el apoyo de la tecnología para la realización del trabajo autónomo, mientras que el menor promedio corresponde al ítem referido a la creación de blogs o medios similares para el desarrollo de las clases. 
Tabla 4

Puntuaciones promedio por ítem del cuestionario aplicado a estudiantes de las carreras vigentes, Facultad de Ciencias Químicas

\begin{tabular}{|lc|}
\hline \multicolumn{1}{|c|}{ ITEMS } & $\begin{array}{c}\text { Puntuaciones } \\
\text { promedio }\end{array}$ \\
\hline $\begin{array}{l}\text { Apoyo de tecnología para la realización del trabajo autónomo (fuera } \\
\text { del aula) }\end{array}$ & 106 \\
\hline Importancia del uso de la tecnología en el aula & 104 \\
\hline $\begin{array}{l}\text { Inclusión de la tecnología en el PEA en el mejoramiento de los } \\
\text { procesos de investigación }\end{array}$ & 104 \\
\hline $\begin{array}{l}\text { Uso de elementos electrónicos en el PEA por parte del cuerpo } \\
\text { docente }\end{array}$ & 104 \\
\hline El uso de dispositivo móvil apoya para el desarrollo de aprendizaje & 100 \\
\hline La calidad de aprendizaje se apoya en el uso de las TIC & 100 \\
\hline La tecnología permite el desarrollo de autonomía en el aprendizaje & 100 \\
\hline $\begin{array}{l}\text { El desarrollo de las asignaturas se facilita con el uso de medios } \\
\text { tecnológicos }\end{array}$ & 98 \\
\hline $\begin{array}{l}\text { La inversión en equipos tecnológicos mejora el rendimiento } \\
\text { estudiantil }\end{array}$ & 97 \\
\hline $\begin{array}{l}\text { Uso permanente de plataforma virtual institucional en el desarrollo } \\
\text { de las asignaturas }\end{array}$ & 96 \\
\hline $\begin{array}{l}\text { Predisposición por parte del sector docente para utilizar elementos } \\
\text { tecnológicos en el PEA }\end{array}$ & 96 \\
\hline El uso de las TIC permite la ejecución de trabajos grupales \\
\hline $\begin{array}{l}\text { El uso de tecnología en el PEA desarrolla mejores relaciones en el } \\
\text { grupo de estudio }\end{array}$ & 95 \\
\hline $\begin{array}{l}\text { Influencia del uso de la tecnología en el nivel de lectura, } \\
\text { procesamiento de información y redacción }\end{array}$ & 93 \\
\hline Fomento de las TIC en habilidades comunicativas & 96 \\
\hline $\begin{array}{l}\text { La utilización de dispositivos tecnológicos en el PEA facilita mejor } \\
\text { comunicación con el cuerpo docente }\end{array}$ & 90 \\
\hline $\begin{array}{l}\text { El profesorado utiliza diversas estrategias innovadoras para motivar } \\
\text { el aprendizaje }\end{array}$ & 86 \\
\hline $\begin{array}{l}\text { Uso exclusivo del pizarrón en el desarrollo de asignaturas de manera } \\
\text { tradicional }\end{array}$ & 83 \\
\hline Necesidad de creación de redes sociales con fines académicos \\
\hline Evaluación de forma tradicional (escrita) & 73 \\
\hline Distracción en el PEA por uso de recursos tecnológicos \\
\hline $\begin{array}{l}\text { Las y los docentes crean blogs o medios similares para el desarrollo } \\
\text { de las clases }\end{array}$ & 67 \\
\hline
\end{tabular}

Fuente: Elaboración propia de la autora (2019) 
A continuación, se presenta la Figura 1, la cual integra los 22 de los 24 ítems, expresados los resultados en porcentajes, según la frecuencia calculada.

Figura 1

Uso de tecnología en el proceso de enseñanza en estudiantes de Facultad de Ciencias Químicas, UCE

\section{Uso de tecnología en el PEA \\ $\square$ SIEMPRE $\square$ CASI SIEMPRE $\square$ OCASIONALMENTE $\square$ NUNCA}

22. El uso de dispositivo móvil apoya para el..

21. El uso de tecnología en el PEA desarrolla.

20. La tecnología permite el desarrollo de.

19. La calidad de aprendizaje se apoya en el uso..

18. Inclusión de la tecnología en el PEA en el..

17. La utilización de dispositivos tecnológicos en..

16. Fomento de las TIC en habilidades..

15. Apoyo de tecnología para la realización del.

14. Distracción en el PEA por uso de recursos.

13. El uso de las TIC permite la ejecución de.

12. El desarrollo de las asignaturas se facilita con.

11. Influencia del uso de la tecnología en nivel de.

10. Evaluación de forma tradicional (escrita)

9. Importancia del uso de tecnología en el aula

8. La inversión en equipos tecnológicos mejora el.

7. Uso exclusivo del pizarrón en el desarrollo de

6. Desarrollo de asignaturas de manera

5. El profesorado utiliza diversas estrategias.

4 .Predisposición por parte del sector docente.

3. Las y los docentes crean blogs o medios.

2. Uso permanente de plataforma virtual

1. Uso de elementos electrónicos en el PEA por.

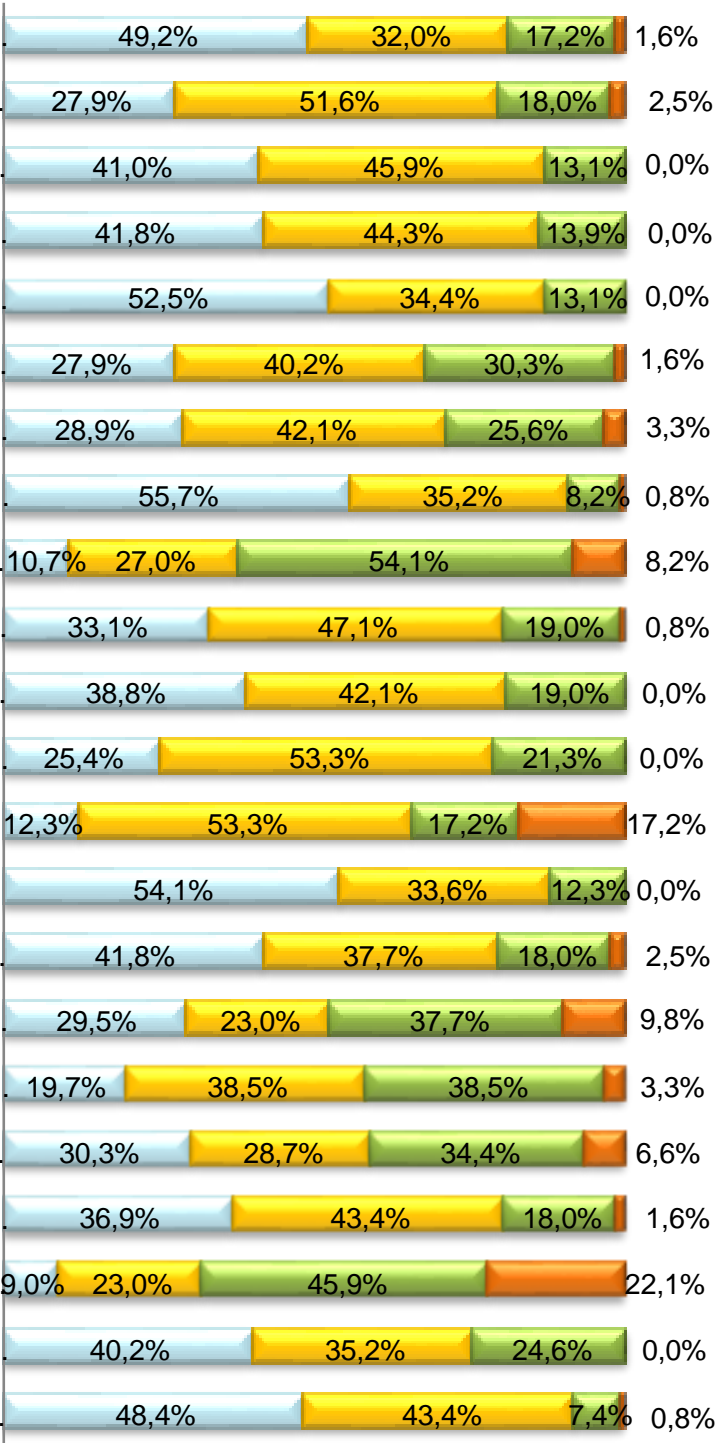

Fuente: Elaboración propia de la autora (2019) con información de encuesta a estudiantes FCQ 


\subsection{Análisis}

Estos resultados permiten establecer el criterio que tiene el estudiantado universitario respecto al uso de la tecnología en su aprendizaje, por lo que se consideró el análisis de cada uno de los ítems, agrupados en las dimensiones establecidas.

La frecuencia sobre el uso de aparatos tecnológicos por el cuerpo docente para el desarrollo de asignaturas, en la figura 1, los resultados se refieren en un $48,4 \%$ que siempre y 43,4\% casi siempre; es decir, en las aulas universitarias estas nuevas herramientas si están mayoritariamente presentes, por tanto, es necesaria la formación al sector docente en el uso de los recursos tecnológicos a fin de alcanzar logros establecidos en el perfil formativo del futuro profesional (Echeverría, 2015). De manera específica, formación en el uso de aplicaciones o softwares de uso didáctico, en la estructuración de distintos recursos de evaluación, esta condición se apoya en el estudio realizado a cerca de 6.000 docentes de 5 países, por Blinklearnig en el 2018 sobre el uso de las tecnologías, señalan que el 46\% no han recibido capacitaciones en el uso de las TIC y que las dos áreas principales de requerimiento formativo son: “- Manejo de software digital (aplicaciones, plataformas, ofimática, etc.). - Búsqueda, edición y producción de contenido digital." (BlinkLearning, 2018, p.53); esto evidencia una falencia importante en el PEA pues, es una realidad que quienes ejercen la docencia y no han tenido formación pedagógica, ponen en riesgo la mala utilización de las TIC como recurso innovador que facilite el aprendizaje al carecer de conocimiento y de tratamiento didáctico de las asignaturas.

En relación con la pregunta sobre el uso de la plataforma virtual institucional para el tratamiento de las cátedras, en la Figura 1, el 40,2\% y 35,2\% de los informantes aseguran que siempre y casi siempre respectivamente, sus docentes hacen uso de la plataforma https://uvirtual.uce.edu.ec que dispone la institución educativa. Sin embargo, en el estudio realizado por Ramirez y Barajas, (2017), se destaca que el 36,2\% de docentes utilizan la plataforma en un nivel bajo, es decir, solo para entregar material o indicar actividades individuales, el 42,38\% por medio de la plataforma aplican exámenes, se comunican con estudiantes, generan trabajos grupales, es decir lo utilizan en un nivel medio, mientras que, apenas un $21,42 \%$ aprovecha el aula para generar informes y realizar procesos de retroalimentación, o sea en nivel alto. Ahora bien, frente a una limitada asignación de fondos públicos, es menester aprovechar los recursos disponibles, como es el caso de la plataforma virtual universitaria, la cual al ser adecuadamente gestionada constituye un espacio para la innovación educativa, las aplicaciones prácticas, fortalecimiento de la creatividad y 
fortalecimiento del aprendizaje colaborativo (Abrigo, 2015; Chamba, 2015 y Delgado, 2009 en Velasteguí, 2017).

En la Figura 1, el cuestionamiento sobre la creación de blogs o medios similares para el desarrollo de clases se establece: $9 \%$ siempre, $23 \%$ casi siempre, $45,9 \%$ ocasionalmente y $22,1 \%$ nunca. Dicha situación evidencia que los blogs no son muy empleados por el personal docente, esto se asemeja al estudio realizado a más de 500 docentes, quienes señalan, en un $72 \%$, que tampoco disponen de blog, canal de Youtube o espacio digital para la interactuación en el PEA (BlinkLearning, 2018); en contradicción con lo señalado en un estudio aplicado a 222 estudiantes, para quienes, los medios de comunicación digital constituyen un medio estimulante en el aprendizaje estudiantil, según Muñoz-Carril, González-Sanmamed y Fuentes-Abeledo (2020).

In parallel, students who are more favourable to using blogs tend to be more likely to use posts as catalysts to stimulate curiosity and imagination, as well as to participate in a playful manner in an enjoyable learning environment $(H 8 ; \beta=0.742 ; p<0.001$; $f 2=1.224)(p .264)$

Esta contraposición descrita, conlleva a plantear la necesidad del cuerpo académico de ser parte de nuevos procesos de aprendizaje sobre los usos pertinentes de las nuevas tecnologías de la información y comunicación, con responsabilidad y ética (García, Godínez y Reyes, 2017).

El siguiente resultado de la Figura 1, el $36,9 \%$ considera que siempre y $43,4 \%$ casi siempre, los docentes tienen predisposición a utilizar elementos tecnológicos en el PEA, lo cual es muy importante, pues se evidencia un interés del sector académico en integrar las TIC al proceso educativo. Hay que resaltar lo señalado por la docente Raquel Aliaga: "Incluir la tecnología en el aula es clave por dos motivos: porque, como docentes, tenemos la obligación de enseñarles a utilizarlas porque serán claves para su futuro laboral; y porque nos ofrece una cantidad ilimitada de recursos educativos (BlinkLearning, 2018, p. 32). romper la primera barrera que constituye la aceptación de medios o recursos como apoyo para la enseñanza y aprendizaje, es una demostración de visualizar a las TIC como herramientas necesarias para que formen parte de la tarea educativa. Las bondades de las TIC señaladas por 533 docentes españoles en orden decreciente son: acceso a mayor número de contenidos y recursos, aprendizaje autónomo, competencia digital y uso 
responsable, aprender a comunicar utilizando diferentes lenguajes o medios de expresión, entre otros (BlinkLearning, 2018).

La consulta realizada sobre estrategias innovadoras para motivar el aprendizaje, por parte de sus docentes, revelo: $30,3 \%$ que siempre, $28,7 \%$ que casi siempre. Este es un indicador que se vincula al ítem anterior y que demuestra el interés del personal académico en la búsqueda permanente de mecanismos para promover el aprendizaje; además que estos generan mayor motivación hacia el aprendizaje, la cual es clave para lograr la apropiación de contenidos, habilidades y fortalecimiento de valores consecuentes con los requerimientos del contexto, así lo confirman Iglesias, Lozano, y Roldán (2018) en un estudio al cuerpo docente:

Se destaca y valora la importancia de seguir formándose permanentemente para garantizar una práctica educativa de calidad (...) La innovación metodológica es esencia y muy importante si no queremos seguir dando clases magistrales y superaburridas donde el alumno no se sienta el protagonista de la misma (p.19)

Entonces, la incorporación de las TIC en Educación Superior está supeditada al interés y compromiso tanto de la docencia como del estudiantado, sin embargo, otro factor decisivo es el que provoca el modelo formativo al que corresponde la carrera y universidad, es decir, tiene una implicación desde la filosofía institucional, pertinencia social, perfiles profesionales, resultados de aprendizajes, contenidos ejecutados a través de la academia, vinculación e investigación, funciones sustantivas de las instituciones universitarias (Zambrano y Zambrano, 2019).

Los resultados sobre el uso exclusivo del pizarrón en el desarrollo de las asignaturas de forma tradicional, en la Figura 1, el 19,7\% indica que siempre, 38,5\% que casi siempre al igual que ocasionalmente, mientras el 3,3\% que nunca; esto permite inferir que, en la docencia aún prevalece la forma tradicional de impartir los contenidos de las asignaturas. Aunque, dada la naturaleza de las profesiones que se forman en la facultad, muchos procedimientos son necesarios que se los demuestre de manera secuencial y procesual. Ahora bien, dar o recibir clases solo usando un medio, puede resultar realmente aburrido, por ello la importancia de la recursividad y diversificación de medios y técnicas en el aula, a fin de generar atención e interés. Cabe añadir la narración verbal de un docente consultado sobre innovación: "Nos adaptamos a las necesidades de los niños y partimos de su interés, 
utilizamos la PDI así como los recursos tradicionales para centrar la atención de los niños: cuentos, canciones, bloques manipulativos, pizarra tradicional,..." (Iglesias et al., 2018, p. 24)

En lo referente a la creación de redes sociales con fines académicos, la información recolectada en la Figura 1 se puede concretar que: 29,5\% estiman que es necesario, 23\% que casi siempre, mientras que $37,7 \%$ que ocasionalmente y $9,8 \%$ que no lo es. Cerca de la mitad del estudiantado opina a favor de la creación de estos mecanismos de comunicación como apoyo a su proceso de aprendizaje. Entre las redes más populares se destaca Facebook, sobre la cual, en un estudio realizado se concluye: “(...) las redes forman parte de un fenómeno global más amplio, y por lo tanto es vital examinar sus implicaciones educativas en un contexto internacional más extenso y explorar si esta situación es recíproca con los entornos educativos" (Hershkovitz y Forkosh Baruch, 2017, p.99)

La consulta sobre la inversión en recursos tecnológicos contribuye en su rendimiento estudiantil, en la Figura 1, señalan en un $41,8 \%$ que siempre, $37,7 \%$ casi siempre, que realizar erogaciones tendientes al equipamiento en tecnología en las instituciones educativas es un factor contribuyente al proceso formativo. El Ministerio de Educación del Ecuador reconoce que, el internet de las instituciones escolares llega generalmente solo al área administrativa, aunque hay interés de su mejoramiento la realidad de las instituciones es lamentable (Diario El Comercio, 2018). De hecho, hay instituciones que requieren la intervención en su infraestructura física, por lo que, la aspiración de contar con infraestructura tecnológica es lejana y peor aún, su inclusión en el desarrollo de los aprendizajes. A nivel universitario, estas dificultades repercuten no solamente en la formación académica del estudiantado, sino en el efecto se irradia hacia la sociedad, los agentes económicos e individuos a través de su proceso productivo de bienes y servicios (Lara, 2015). Bien se afirma que:

El desarrollo de la ciencia, la tecnología y la innovación constituye un factor indispensable para promover el progreso socioeconómico de la sociedad moderna. En consecuencia, lograr el cumplimiento de este fin requiere de procedimientos asociados con la formulación de políticas públicas que contribuyan al desarrollo y bienestar de la sociedad, cuyo eje sea el conocimiento sustentado en los aportes de la investigación científica, el avance tecnológico y la apropiación de las innovaciones (Saltos, Pelegrín, y Esquivel, 2017) 
Según datos oficiales durante el 2016, en Ecuador la inversión tecnológica alcanzó el $1,88 \%$ del PIB, alrededor de $\$ 1.900$ millones, cifra todavía insuficiente para que las nuevas tecnologías sean permanentemente parte del proceso docente educativo (Diario El Telégrafo, 2016). Cabe añadir frente a esta realidad, un dato del 2017:

Cuatro universidades, que juntas en ese momento llegaban a tener 1.238 estudiantes, recibieron un trato privilegiado política y económicamente; mientras que 28 universidades y escuelas politécnicas públicas que acogían a más de 250 mil estudiantes percibieron niveles de ingreso en términos relativos" (Plan V, 2017, párr.23)

Así, un estudiante de una de estas 4 universidades, recién creadas, recibió tres veces más (\$13.705) que uno de una institución pública como la Universidad Central (4.097), situación por demás difícil para lograr una formación integral.

En este sentido de limitación de recursos, los hogares ecuatorianos han realizado inversiones particulares para la adquisición de computadoras; así se evidencia en la Tabla 5, una evolución significativa del 2012 al 2016, aunque hay decrecimiento en el 2018.

Tabla 5

Ecuador: Variación de la participación de los hogares en equipamiento de computadoras portátiles y de escritorio. 2012, 2016, 2018

\begin{tabular}{|lcc|c|c|c|}
\hline \multicolumn{1}{|c}{ Equipamiento } & 2012 & 2016 & Variación & 2018 & Variación \\
\hline Computadora portátil & $13.9 \%$ & $27,6 \%$ & $13,7 \%$ & $24,2 \%$ & $-3.4 \%$ \\
\hline Computadora de escritorio & $26.4 \%$ & $26.7 \%$ & $0,3 \%$ & $24,5 \%$ & $-2.2 \%$ \\
\hline
\end{tabular}

Fuente: Elaboración propia a partir de la información del INEC $(2013,2016,2018)$

En lo referente a la relevancia del uso de la tecnología en el aula, los informantes del sector estudiantil señalan, en un $54,1 \%$ que siempre, 33,6\% casi siempre. Lo anterior evidencia que, las herramientas innovadoras al ser integradas en el aula son apreciadas como beneficiosas. Por eso se destaca que, la forma y alcance de la implementación de las TIC en los centros educativos influirá en el impacto de su utilización, de dos maneras; facilitando o restringiendo su acceso, específicamente en lo referente a estudiantes que poseen una brecha digital o escasas posibilidades de conexión a medios digitales, de ser así, más bien se constituye en un factor de desigualdad. (Hermosa, 2015).

Por tanto, a fin de aprovechar las potencialidades de las TIC, se requiere la consideración como política educativa, no solo en la designación de recursos, sino en el 
alcance y cobertura entre todo el alumnado, de manera que su uso sea equitativo y favorecedor y no un factor más de discriminación en el proceso. A propósito García, Godínez, y Reyes (2017), basados en la Conferencia Mundial sobre la Educación Superior recomiendan:

En un proceso de enseñanza-aprendizaje juegan un papel importante las tecnologías de la información y comunicación como apoyo en la interacción con actividades didácticas que integran lo visual, novedoso e interactivo; incentiva el uso de aplicaciones, plataformas y redes sociales; promueve nuevas formas de enseñanza; facilita la búsqueda de información y comunicación, el desarrollo de actividades prácticas del quehacer docente. (p. 6)

Sin embargo en contraposición a la apreciación estudiantil descrita, en un estudio realizado al profesorado sobre su aceptación frente a la implementación de las TIC en los centros educativos alcanzó un 58\%, lo que indica que aún se deben vencer filosofías anteriores.

En lo que concierne a los resultados expuestos en la Figura 1, sobre la realización de las evaluaciones de manera tradicional, es decir, escritas con papel y lápiz, la población establece, en un $12.3 \%$ siempre, $53,3 \%$ casi siempre y $17,2 \%$ ocasionalmente y nunca. Lo anterior denota que, quienes hacen docencia aún utilizan de manera exclusiva estos recursos a la hora de realizar las evaluaciones, negándose la oportunidad de diversificar con otros recursos al momento de valorar en sus discentes los logros alcanzados. Aunque también, en evaluaciones en línea se corre el riesgo de estructurar pruebas tipo test sin base pedagógica, alineadas desde el software tecnológico y muy distante del modelo pedagógico institucional. Esto deberá ser superado a través de la estructuración de diversas técnicas de evaluación, tanto presenciales como en línea, que impliquen todos los ámbitos del conocimiento: conceptuales, procedimentales y actitudinales.

Sobre el cuestionamiento de la influencia del uso de la tecnología en el nivel de lectura, procesamiento de la información y redacción, el alumnado responde: 25,4\% siempre, y $53,3 \%$ casi siempre. En relación con lo anterior, según especialistas, las tecnologías de información aplicadas en educación, constituyen:

(...) un fenómeno de gran trascendencia social, son un medio para potenciar la educación a partir del cual se puede fortalecer el proceso de lectura-escritura, dado que los estudiantes son hoy más sensibles a un entorno digital, porque posibilita un 
mayor grado de interacción con dispositivos electrónicos, teléfonos móviles, televisión digital, videojuegos y el uso habitual del internet. (Hermosa, 2015, p. 123)

De igual manera, en un estudio realizado a 24 estudiantes, sobre la aplicación de herramientas tecnológicas para desarrollar habilidades de lectoescritura y de procesamiento de información, se determinó que 14 , es decir 58\%, alcanzaron una nota superior a siete sobre diez (Suárez, Pérez, Vergara, y Alférez, 2015). En Ecuador, una realidad alarmante son los resultados arrojados en las evaluaciones de las pruebas PISA de 2017 señalan que: “29\% de los jóvenes de 15 años alcanzó el nivel mínimo de competencia en Matemática, $43 \%$ en Ciencias y $49 \%$ en Lectura" (Tamayo-Madrid, 2019), citando a Torres (2019); lo que demuestra la importante necesidad del desarrollo de esta habilidad, pues, a partir de la lectura comprensiva se facilita el aprendizaje de otras disciplinas,

En la Figura 1 sobre el uso de medios tecnológicos como facilitadores del avance de las asignaturas, se obtiene: el $38,8 \%$ y $42,1 \%$ responden que siempre y casi siempre. Al respecto, investigadores de la Universitat Oberta de Catalunya afirman:

(...) la integración adecuada de las Tecnologías de la Información y la Comunicación (TIC) en el diseño curricular de las asignaturas cuantitativas aplicadas favorece una mayor dosis de participación, creatividad, aprendizaje significativo y motivación por parte del estudiante. Esto permite establecer (...) objetivos más ambiciosos y afrontar el análisis de problemas más realistas y complejos. (Juan, Huertas, Steegmann y Terrádez, 2006, parr. 4)

En relación al ítem sobre el uso de las TIC para la ejecución de trabajos grupales, en la Figura 1 se destaca: 33,1\% señalan que si permite, y el 47,1\% casi siempre. Estos resultados son confirmados con la investigación realizada sobre el tema:

(...) el análisis de distintas opiniones en el sector educación, avalan la importancia y creciente perspectiva de la tecnología, lo que fomentaría un aprendizaje social y colaborativo, con una vertiente capaz de generar una vinculación de una educación transformadora y adaptable a las sociedades actuales. (Hernández, 2017, p.332)

Dichas aseveraciones concuerdan que, la conformación de grupos sincrónicos de trabajo es un elemento favorecedor del PEA, ya que visibilizan la capacidad de gestión de las TIC por parte del estudiantado, pero, la efectividad está limitada por la capacidad 
planificadora y organizativa por parte de docentes. Por ello, se afirma que los ambientes colaborativos propician la igualdad, información bidireccional, retroalimentación y motivación al aprendizaje personal (González, 2017).

En la Figura 1, sobre la consideración de los recursos tecnológicos como un elemento distractor en el proceso educativo, las personas investigadas indican: en un $54,1 \%$ ocasionalmente y $8,2 \%$ nunca, es decir, mayoritariamente el estudiantado afirma que las herramientas tecnológicas no causan distracción en su proceso de aprendizaje. Sin embargo, este criterio difiere respecto del sector docente, pues en un estudio planteado, el $72 \%$ del personal de enseñanza, consideran al uso de las TIC como más adecuado para un uso de nivel social o lúdico antes que académico, por lo que, provocan distracción, dispersión y pérdida de tiempo (Sanabria y Hernández, 2011). Bajo este argumento, es común que se impida el ingreso e integración en el aula artefactos como teléfonos inteligentes, IPad, Portátiles. Sobre esto se sostiene:

Los jóvenes actuales viven con la tecnología a cuestas notebooks, celulares que forman parte del paisaje de las aulas de clases, por otro lado docentes viejos y jóvenes, utilizan las Tic's como recursos para el aprendizaje, lo que no siempre funciona eficientemente ya que estos recursos antiguos o modernos pueden transformarse en distractores contrarios al aprendizaje, sumado a que entre docentes y estudiantes hay una gran brecha tecnológica. (Pasto, 2015, p. 22). citando a Yankovic, (2013)

En contraposición sobre esta percepción, en el 2018 los resultados obtenidos en una investigación a 5.977 docentes, apenas el 13\% estima que el uso de tecnología reduce la atención en el PEA. (BlinkLearning, 2018) 
Figura 2

Horas diarias destinadas para uso de TIC por estudiantes Facultad de Ciencias Químicas

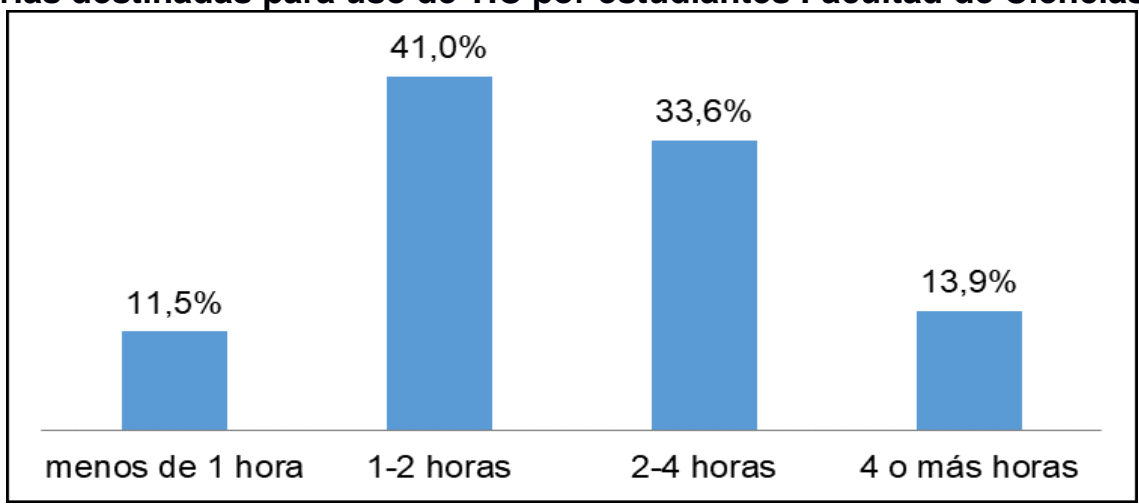

Fuente: Elaboración propia de la autora (2019), con información de encuesta a estudiantes FCQ

La Figura 2, presenta el tiempo de uso de TIC por parte de la población estudiantil, la cual muestra que el 74,6\% utiliza diariamente las TIC entre 1 y 4 horas. En relación con aprendizaje autónomo que debe cumplir el alumnado de la Universidad Central, es de 6 horas diarias en promedio; este tiempo es según el cálculo de organización curricular asumido por la institución, es decir, 1,5 horas de aprendizaje autónomo por cada hora de docencia. Entonces, el tiempo uso de las TIC indicaría que las tareas o actividades de las asignaturas no implican el uso de herramientas tecnológicas, o en su defecto, la dificultad de accesibilidad a herramientas de conectividad.

Es importante resaltar que, el INEC señala que en el 2016, a nivel nacional, el 55,6\% de las personas han destinado el internet a diversas actividades, como fuente de información el 38\%, como medio de educación y aprendizaje el $23,2 \%$ y para comunicación el 31,5\% (INEC, 2016). Asimismo, a nivel nacional, en el 2018, el 55,9\% de la población utiliza el internet mientras que la frecuencia de uso al menos una vez al día, en un $84 \%$, de igual manera, los hogares tienen acceso internet en un 37,2\% (INEC, 2018). Estos datos muestran cómo la tecnología está inundando en todos los sectores y niveles significativamente, pero su utilización para el aprendizaje se ve reducida.

Sobre el aporte de la tecnología al aprendizaje autónomo, en la Figura 1, los informantes responden: en un 55,7\% y 35,2\% que siempre y casi siempre respectivamente, esta contribuye al desarrollo de actividades independientes. Sobre este aspecto, Rubio (2013) afirma:

El Internet como red sin restricciones de tiempo o espacio ofrece un mundo de información capaz de ofrecer al estudiante una amplia variedad de contenidos, razón por la cual el aprendiz debe aprender a elegir el procedimiento de autoaprendizaje y la 
autoformación, y es que al tener libre acceso a la información adquirida en tiempo real, aparecen también nuevas implementos útiles que contribuyen al incremento del proceso de enseñanza-aprendizaje, a través de la comunicación mediante foros, chats, videoconferencia, plataformas educativas, páginas web, bases de datos, aplicaciones, etc. (p. 104)

El planteamiento anterior, comparado con el estudio realizado por Climént (2018), sobre las actividades rutinarias realizadas por el alumnado destaca: “(..) el promedio de horas dedicado semanalmente a las redes sociales (10.2 horas) es superior al destinado al entretenimiento (9 horas), al estudio (7.7 horas), al deporte (7.3 horas) y a las tareas domésticas (6.3 horas)." (p.17), lo cual permite deducir que no siempre las horas de uso de tecnología están vinculadas con el aprendizaje autónomo que deben dedicar fuera de la jornada académica.

Lo señalado por el sector estudiantil, sobre el fomento de las TIC en las habilidades comunicativas, en la Figura 1 el $28,9 \%$ indica que siempre y $42,1 \%$ casi siempre si aportan para el mejoramiento de la comunicación por los procesos interacción; lo cual es una particularidad de las herramientas tecnológicas. Es decir, el estudiantado al tener espacios abiertos y flexibles de actuación estimula su participación en comunidades de aprendizaje colaborativo, siendo el principal instrumento, el lenguaje. De ahí que, deberá ocurrir una necesaria transformación de las prácticas educativas, mediante inclusión de proyectos coparticipativos, tendientes a la utilización de las TIC, de forma que se generen mejores canales de comunicación e información, no solo en su interacción educativa, sino en la formación profesional (Basilotta, García y López, 2014).

En lo referente al uso de medios tecnológicos, como elementos que facilitan el diálogo pedagógico y arbitran la relación docente y discente, en la Figura 1, se evidencia que el $27,9 \%$ y $40,2 \%$, siempre y casi siempre respectivamente, si los valoran en el proceso de interacción con el personal académico. Alrededor de ello, en un estudio realizado a grupos estudiantiles de tres universidades ecuatorianas, se concluye que: "La vinculación es mayor cuando el docente convierte a los medios sociales en mecanismos metodológicos de aprendizaje, los medios sociales sirven como espacios para propiciar las tutorías virtuales ya que son entornos familiares e interactivos para los alumnos." (Abel, Arrobo-Agila, y Carrión, 2018, p. 248). Aunque se puede afirmar que, la educación tradicional universitaria se ve afectada por la poca accesibilidad o apertura por parte del profesorado, pues existe el recelo 
a perder su autoridad, por lo que adopta una posición de superioridad y distancia con el estudiantado.

En la Figura 1, sobre la contribución de la tecnología en la investigación definen, en un $52,5 \%$ que siempre y $34,4 \%$ casi siempre. Es un hecho que, el mayor medio utilizado para procesos de búsqueda de información y generación de conocimiento se lo alcanza en las diversas formas de difusión científica, la cual en la actualidad fundamentalmente, es compartida por medio de los recursos digitales. Gracias a esta transmisión se nutre el conocimiento, la investigación, la innovación y la tecnología. En este sentido, las dos carreras vigentes, de Bioquímica y Farmacia y de Química, en su estructura formativa están significativamente ancladas al campo de investigación analítica, correlacional y experimental, por esta razón, es necesario el fortalecimiento de las habilidades investigativas planteadas en el perfil de egreso. Siendo, el mecanismo adecuado la integración pedagógica de las TIC y la superación de las barreras extrínsecas del sistema educativo universitario, respecto de la provisión de recursos técnicos y tecnológicos.

Es así que, la Educación Superior, responsable de la formación integral, debe promover estrategias para fortalecer la sociedad del conocimiento, a través de las TIC y la investigación como requerimiento para la innovación tecnológica (Licona y Veitya, 2019). Sin embargo, en los últimos tiempos, en el sistema universitario ecuatoriano se evidencia todavía insuficiente contribución en recursos para los procesos investigativos. Básicamente, el alcance de las habilidades investigativas, en la Facultad de Ciencias Químicas, en un estudio realizado se sintetizan de manera que:

“(..) se integran y se sintetizan en la habilidad de observación, recolección y organización de la información, uso de métodos de análisis de información, evaluación de métodos de investigación de acuerdo con el objeto de estudio, empleo de métodos de medición, de abordaje de problemas, manipulación de objetos y su registro, de interpretación y explicación, de predicción, interpretación de datos estadísticos, de experimentación, de discusión de resultados, argumentación y el uso del tiempo y el espacio." (Espinel y Calderón, 2019, p.41)

En lo referente a la calidad del aprendizaje con apoyo del uso de las TIC, la Figura 1 muestra que: el $41,8 \%$ señala que siempre y 44,3\% casi siempre, la integración de la tecnología apoya la calidad de aprendizaje. Este criterio, es apoyado por los resultados de un estudio realizado en España, sobre el impacto de las TIC, en el que los investigadores 
concluyen: “(...) las TIC actúan como un factor dinamizador de los procesos de enseñanzaaprendizaje, propiciando transformaciones respecto a los roles docentes-discentes" (Colás, De-Pablos y Ballesta 2018, p.20). Por ello, su aporte contribuye en la formación en los futuros profesionales en lo referente a conocimientos, capacidades, competencias y habilidades, requerimientos para una adecuada participación en esta compleja y emergente sociedad de la información y el conocimiento,

Sobre el aporte de las TIC en el desarrollo de autonomía de aprendizajes, el 41\% consideró que siempre y el 45,9\% casi siempre. Estos resultados son avalados por las conclusiones del estudio de Colás et al. (2018) sobre la incidencia de las TIC en el aula en la transformación del estudiante: "(...) se propicia que el alumno tenga un papel más activo en su proceso de aprendizaje, así como una mayor autonomía y responsabilidad en los procesos de toma de decisiones, a la mediación de las TIC en los procesos de enseñanza y aprendizaje (...)" (p.20). Esta autonomía se construye en un ambiente con determinadas condiciones, con recursos y herramientas apropiadas y mediante procesos cuidadosamente planificados y con importante interacción docente, pues no cualquier actividad en el aula conduce a la autonomía.

La consulta respecto de que la tecnología genera mejores relaciones entre el grupo de estudio, un $27,9 \%$ considera que siempre y $51,6 \%$ casi siempre. Estos resultados se apoyan en lo señalado por García, et al. (2017), sobre esta nueva forma de hacer educación:

Las transformaciones a la luz de las nuevas tecnologías han cambiado de manera reveladora las relaciones humanas, las nuevas formas de acceso a las tecnologías de la información y comunicación son ahora el motor de los nuevos paradigmas respecto de cómo se relacionan las personas con nuevas formas de participación social, de control y activismo a través de las redes sociales. (p. 15)

Los educandos, al respecto del uso de su dispositivo móvil (celular) apoya en el desarrollo de su aprendizaje, los resultados determinan en un 49,2\% que siempre y $32 \%$ casi siempre; esto es corroborado por el INEC (2018) que señala, que del grupo etario entre 1624 años, asociado a la edad estudiantil universitaria, el 71,7\% dispone de un celular activado y de ellos, el 63,2\% corresponde a un teléfono inteligente (Smartphone), lo cual permite la accesibilidad a la información necesaria para desarrollar actividades académicas. 
Figura 3

Formas de acceso a internet del estudiantado Facultad de Ciencias Químicas

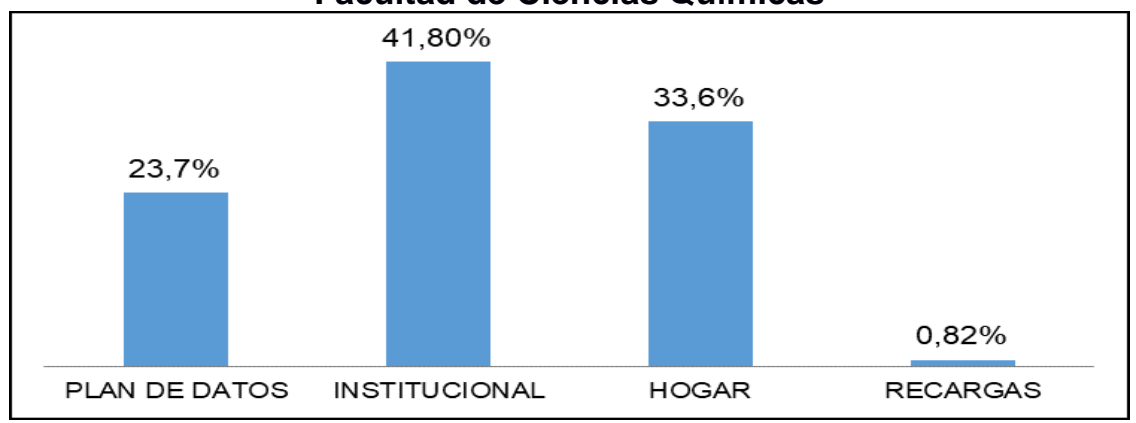

Fuente: Elaboración propia de la autora (2019) con información de la encuesta a estudiantes FCQ

En la Figura 3, se evidencia en forma mayoritaria que, el sector estudiantil universitario accede al internet facilitado por la institución educativa, en segundo lugar por la red desde su hogar, y casi una cuarta parte, tiene plan de datos contratado, esto muestra la condición económica estudiantil de universidades del sector público. Asimismo, por las condiciones de horarios y requerimientos académicos, el estudiantado de la Facultad no puede realizar una actividad laboral paralela para mejorar su situación económica, lo que limita el acceso mediante la contratación de planes de datos. Aunque lo anterior, manifiesta discrepancia con los resultados a nivel nacional, los cuales señalan que el medio de conexión a internet en primer lugar es del hogar y en tercero de la institución educativa, siendo, el grupo etario de 16-24 años, en un 81,19\% que mayormente utiliza el internet (INEC, 2018). Esta realidad del estudiantado de la institución, es afirmada por el sector docente, que establece cierto grado de dificultad al momento de trabajar con medios tecnológicos que requiere utilizar el plan de datos, pues, lo dispone un número limitado de estudiantes.

\section{Conclusiones}

Las sociedades en vías de desarrollo no encontrarán un avance efectivo mientras no se asuma la educación con responsabilidad. Es evidente que, lo que se ha hecho hasta ahora es transmitir y sostener el modelo económico dominante. Dicha transformación no está sostenida en la mayor cantidad de recursos, ni en la adquisición de hardware y software exclusivamente, sino en la adopción de una nueva mentalidad por parte del cuerpo docente para que miren las TIC como recursos importantes para ser incorporados en el aula, porque apoyan y estimulan un mejor aprendizaje. Ello requiere que, la docencia desaprenda las viejas prácticas tradicionales para dar paso a formas pedagógicas, más innovadoras y 
desarrolladoras para el ser humano, el mismo que necesita estar dotado de competencias, capacidades y habilidades tendientes al saber, saber hacer, saber actuar, pero sobre todo saber ser e interactuar con sus pares.

En virtud de los resultados obtenidos es importante destacar el rol del personal docente, pues tiene en sus manos la responsabilidad de una formación integral, tomando en cuenta la multidimensionalidad del estudiantado, así como los requerimientos de una sociedad que es más demandante, pues el aporte de futuros profesionales no se circunscribirá a aplicaciones de los contenidos, sino a ser transformadores efectivos de lo establecido.

Se ha demostrado el criterio estudiantil respecto de las contribuciones de las TIC en su proceso formativo, específicamente en el fortalecimiento de sus habilidades comunicativas, investigativas, operativas, de mejor interacción. Así también, se manifiesta la contribución de los medios tecnológicos en el alcance de los objetivos formativos profesionales, el apoyo al aprendizaje autónomo, trabajo grupal, al mejoramiento en los procesos intrapersonales e interpersonales de aprendizaje

El sector estudiantil considera la incorporación de las TIC en el aula como positiva, sobre todo, porque aún son evidentes las prácticas docentes eminentemente tradicionales en el desarrollo de las asignaturas y evaluación. Por ello, el cuerpo docente debe superar los prejuicios respecto del uso de la tecnología en el PEA.

El dispositivo móvil más común en el estudiantado y mayormente utilizado, con fines académicos, es el celular, ya que mediante su uso le permite mejor accesibilidad. En este sentido, la accesibilidad está posibilitada, de manera más amplia, por la conexión a la red institucional, situación representada por la condición económica del sector estudiantil universitario.

También se rescata que el $74 \%$ de las personas discentes dedican entre 1 y 4 horas diarias al uso del internet, sin embargo, sería deseable que este tiempo estuviera relacionado con actividades académicas.

El presente estudio permitió evidenciar el criterio sobre el uso y el aporte de la tecnología en el aprendizaje del estudiantado de la Facultad de Ciencias Químicas de la Universidad Central del Ecuador, destacando que ésta, si se encuentra integrada de forma positiva en los procesos áulicos mas no es generalizada. Por lo que, se requiere una mayor y mejor integración en el PEA, para que se constituya en herramienta motivadora y generadora de mejores procesos de apropiación. De esta manera y en virtud de las ventajas señaladas a 
nivel de otras investigaciones, se aprovecharán las potencialidades que ofrecen las TIC al proceso docente educativo; pero para ello se requiere, una adecuada planificación curricular, en la que se establezca con especificad la forma y el alcance que se otorgará a las TIC, así como los mecanismos de ejecución que deberán articular los elementos curriculares (resultados de aprendizaje, metodología y evaluación).

El uso de medios tecnológicos por parte del sector estudiantil de las carreras de Bioquímica y Farmacia y la de Química permiten desarrollar actividades de docencia, aprendizaje colaborativo y aprendizaje autónomo. Sin embargo, la búsqueda de un impacto positivo de las TIC también debe estar orientada al pensamiento crítico y creativo, de manera que se generen actividades y tareas de calidad, por ello es menester, el desarrollo del trabajo autónomo como forma de lograr procesos de autocapacitación y reflexión de los contenidos.

Las nuevas tecnologías constituyen una gran oportunidad para el proceso educativo, por tanto, actores e instituciones educativas tienen la responsabilidad de prepararse para una utilización pertinente y para generar espacios cooperativos y colaborativos en la construcción de aprendizajes significativos y útiles para un desenvolvimiento efectivo en la sociedad.

Hay que evitar el criterio de que la educación debe adaptarse a la tecnología, si no lo contrario, la tecnología debe ser integrada al proceso educativo como herramienta para el alcance de habilidades y capacidades. Por ello, las instituciones están obligadas a capacitar a quienes hacen docencia y a conducir al estudiantado a aprovechar la accesibilidad a recursos digitales.

Las instituciones de educación superior muestran importantes debilidades en el ámbito pedagógico, vinculadas con la escasa formación por parte del sector docente en esta área del conocimiento, siendo necesario que previo a la incorporación del personal novel deberían asegurarse de la formación en pedagogía o docencia universitaria y la verificación del idóneo conocimiento, adecuación y utilización de TIC en la gestión de aula; mientras que, para los docentes de planta es necesario promover mayor y mejor capacitación sobre las TIC.

\section{Agradecimientos}

Al estudiantado de segundo semestre de las Carreras de Bioquímica y Farmacia, y de Química, período académico marzo-agosto 2019, por su participación en la recolección de datos. 


\section{Referencias}

Abel, Suing., Arrobo-Agila, Juan. y Carrión, Nelson. (2018). Redes Sociales y su interacción entre docentes $y$ estudiantes: caso Ecuador. Recuperado de https://www.researchgate.net/publication/323444375 REDES SOCIALES Y SU INTE RACCION ENTRE DOCENTES Y ESTUDIANTES CASO ECUADOR

Baptista, Pilar, Collado, Carlos, y Hernández, Roberto. (2014). Metodología de la Investigación. México: McGrawHill.

Basilotta, Verónica., García, Ana. y López, Camino. (2014). Las TIC en el aprendizaje colaborativo en el aula de Primaria y Secundaria. Comunicar, 21(42), 65-74. https://doi.org/10.3916/C42-2014-06.

https://www.google.com/search?q=Las+TIC+en+el+aprendizaje+colaborativo+en+el+a ula+de+Primaria+y+Secundaria\&oq=Las+TIC+en+el+aprendizaje+colaborativo+en+el+ aula+de+Primaria+y+Secundaria\&aqs=chrome..69i57j69i60.2135j0j8\&sourceid=chrom e\&ie=UTF-8

BlinkLearning. (2018). IV Estudio sobre el uso de la tecnología en la educación. Informe de resultados Chile Septiembre 2018 España . Colombia . México · Perú . Chile. BlinkLearning. Recuperado de https://www.realinfluencers.es/wpcontent/uploads/2018/11/iv-estudio-tic-2018-chile.pdf

Consejo de Educación Superior-CES. (2013). Reglamento de Régimen Académico. Recuperado de http://www.ces.gob.ec/doc/Reglamentos_Expedidos_CES/codificacin del reglamento de rgimen acadmico.pdf

Consejo de Educación Superior-CES. (2019). Reglamento De Régimen Académico. Recuperado de: https://doi.org/DOI 10.1111/j.1438-8677.2009.00206.x

Climént, Juan Bautista. (2018). Factores adversos al fomento de hábitos de estudio y aprendizaje en educación superior: Un estudio de caso. Actualidades Investigativas en Educación, 18(3), 255-284. Doi https://doi.org/10.15517/aie.v18i3.34122

Colás, María Pilar., De Pablos, Juan. y Ballesta, Javier. (2018). Incidencia de las TIC en la Enseñanza En El Sistema Educativo Español: Una Revisión de La Investigación. Revista de Educación a Distancia (RED), 18(56), Recuperado de https://revistas.um.es/red/article/view/321471

La tecnología y los servicios se integran en plan de infraestructura educativa. (29 de mayo, 2018). Diario El Comercio. Recuperado de https://www.elcomercio.com/actualidad/tecnologia-servicios-mejoramientoinfraestructura-educacion.html

Ecuador invierte el 1,88 del PIBB en tecnologia einnovacion. (25 de noviembre, 2016). Diario El Telégrafo. Recuperado de https://www.eltelegrafo.com.ec/noticias/sociedad/6/ecuador-invierte-el-1-88-del-pib-entecnologia-e-innovacion

Durán, Beatriz., Barragán, Jorge., González, Juan., y Guzmán, Teresa. (2017). Formación en 
TIC y competencia digital en la docencia en instituciones públicas de educación superior. Apertura (Guadalajara, Jal.), 9(1), 80-96. https://doi.org/10.18381/ap.v9n1.922

Echeverría, Ana. (2015). Uso de las TIC en la Docencia Universitaria: Opinión del Profesorado de Educación Especial. Actualidades Investigativas en Educación, 14(3). Recuperado https://revistas.ucr.ac.cr/index.php/aie/article/view/16131/18103\#autor 1No

Espinel, Elithsine. (2015). Guía metodológica para la elaboración del plan e informe de Investigación: Para trabajos de grado y posgrado. Quito: Servicios Publicitarios.

Espinel, Elithsine y Calderón, Mariana. (2019). Investigación y difusión científica: Competencias esenciales en la formación universitaria del profesional en Ciencias Químicas. Tsafiqui, 12(1), 37-52. https://doi.org/10.29019/tsafiqui.v12i1.555

Gallent, Cinta., y Tello, Isabel. (2017). Percepción del alumnado de traducción de la Universidad Internacional de Valencia (VIU) sobre el ciberplagio académico. Revista Digital de Investigación en Docencia Universitaria, 11(2), 90-117. https://doi.org/10.19083/ridu.11.563

García, María., Godínez, Guadalupe., y Reyes, Joaquín. (2017). Las Tic en la educación superior, innovaciones y retos. Revista Iberoamericana de Las Ciencias Sociales y Humanísticas., 6(12). Doi 10.23913/ricsh.v6i12.135

Gavilanes, Marco., Yanza, William., Inca, Alex., Torrez, German. y Sánchez, Roberto. (2019). Las TICs en los procesos de enseñanza y aprendizaje. Ciencia Digital, 3(2.6). Doi https://doi.org/10.33262/cienciadigital.v3i2.6.575

González, Denis. (2017). Ambientes colaborativos virtuales para el aprendizaje individual. Actualidades Investigativas en Educación 17(2). Recuperado de https://revistas.ucr.ac.cr/index.php/aie/article/view/28092

Gros, Begoña. (2016). Retos y tendencias sobre el futuro de la investigación acerca del aprendizaje con tecnologías digitales. Revista de Educación a Distancia (RED), (50). Doi https://doi.org/10.6018/red/50/10

Hermosa, Paola. (2015). Influencia de las tecnologías de información y comunicación (TIC) en el proceso enseñanza-aprendizaje: una mejora de las competencias digitales. Revista Científica General José María Córdova, 13, 121-132. Recuperado de http://www.scielo.org.co/pdf/recig/v13n16/v13n16a07.pdf

Hernandez, Ronald. (2017). Impacto de las TIC en la educación: Retos y Perspectivas. $\begin{array}{lllll}\text { Propósitos } \quad y & \text { Representaciones, } & 5(1) & \end{array}$ https://dialnet.unirioja.es/servlet/articulo?codigo=590476

Hershkovitz, Amon. y Forkosh Baruch, Alona. (2017). La relación profesor-alumno y la comunicación en Facebook: percepciones de los alumnos. Comunicar: Revista Científica Iberoamericana de Comunicación y Educación, (53). Recuperado de https://recyt.fecyt.es/index.php/comunicar/article/view/62671 
Iglesias, Marcos, Lozano, Inés y Roldán, Irene. (2018). La calidad e innovación educativa en la formación continua docente: un estudio cualitativo en dos centros educativos. Revista Iberoamericana de Educación, 77, 13-34.

Instituto Ecuatoriano de Estadísticas y Censos (INEC). (2013). Tecnologías de la Información y Comunicación-TIC. Recuperado de https://www.ecuadorencifras.gob.ec/tecnologiasde-la-informacion-y-comunicacion-tic/

Instituto Ecuatoriano de Estadísticas y Censos (INEC). (2016). Tecnologías de la Información y Comunicación. Recuperado de https://www.ecuadorencifras.gob.ec/documentos/webinec/Estadisticas Sociales/TIC/2016/170125.Presentacion Tics 2016.pdf

Instituto Ecuatoriano de Estadísticas y Censos (INEC). (2017). Tecnologías de la Información y Comunicación ENEMDU - TIC. Recuperado de https://www.ecuadorencifras.gob.ec/documentos/webinec/Estadisticas Sociales/TIC/2017/Tics 2017 270718.pdf

Instituto Ecuatoriano de Estadísticas y Censos (INEC). (2018). Tecnologías de la Información y Comunicación. Recuperado de https://www.ecuadorencifras.gob.ec/documentos/webinec/Estadisticas Sociales/TIC/2018/201812 Principales resultados TIC Multiproposit $\underline{\text { o.pdf }}$

Juan, Ángel., Huertas, María., Steegmann, Cristina. y Terrádez, Manuel. (2006). Uso e integración de las TIC en asignaturas cuantitativas aplicadas: la experiencia de los estudios de informática y multimedia de la UOC. Education in the Knowledge Society (EKS). Recuperado https://campus.usal.es/ teoriaeducacion/rev numero 07/n7 art juan huertas steegm an terradez.htm

Lara, Juan. (2015). Impacto de la inversión en educación, ciencia y tecnología en el desarrollo económico de América Latina y el Caribe: 1995 - 2014. Gestiopolis. Recuperado de https://www.gestiopolis.com/impacto-inversion-educacion-cienciatecnologia-desarrollo-economico-america-latina-caribe-1995-2014/

Licona, Karuba. y Veitya, María. (2019). La Formación en y para la investigación y el uso de las Tecnologías de la Información y Comunicación. Revista Atenas, 2(46). Recuperado de https://atenas.reduniv.edu.cu/index.php/atenas/article/view/470/737

Muñoz-Carril, Pablo., González-Sanmamed, Mercedes., y Fuentes-Abeledo, Eduardo. (2020). Use of blogs for prospective early childhood teachers. Educacion $X X 1,23(1)$, 274-273. https://doi.org/10.5944/educxx1.23768

Pasto, Irma. (2015). Uso de las tecnologías de la información y comunicación y el aprendizaje logrado por los estudiantes del centro de educación básica, ciudad de Ventanas, en el cantón Ventanas, Provincia de Los Ríos. Universidad Técnica de Babahoyo. Recuperado de http://dspace.utb.edu.ec/bitstream/49000/1684/1/T-UTBCEPOS-MDC-0000031.pdf 
Plan V. (18 de septiembre, 2017). La encrucijada económica de la universidad ecuatoriana. Recuperado de https://www.planv.com.ec/historias/sociedad/la-encrucijada-economicala-universidad-ecuatoriana

Poveda, Miguel, y Thous, María del Carmen. (2013). Mundos virtuales y avatares como nuevas formas educativas. Ilu. https://doi.org/10.5209/rev HICS.2013.v18.44262

Ramirez, Wilmer. y Barajas, Juan. (2017). Uso de las plataformas educativas y su impacto en la práctica pedagógica en instituciones de educación superior de San Luis Potosí. Edutec. Revista Electrónica de Tecnología Educativa, (60). Doi https://doi.org/10.21556/edutec.2017.60.798

Rodríguez, María. (2005). Aplicación de las TIC a la evaluación de alumnos universitarios. Education in the Knowledge Society (EKS), 6(2). Recuperado de http://campus.usal.es/ teoriaeducacion/rev numero $062 / n 602$ art rodriguez conde. $\underline{\mathrm{htm}}$

Rubio, Catalina. (2013). Canales y accesos a la información que almacenan y difunden contenidos digitalizados. Las TIC en educación. Escritos en la Facultad, 9(84), 104. Recuperado de https://fido.palermo.edu/servicios dyc/publicacionesdc/vista/detalle articulo.php?id libr $\underline{0=443 y i d}$ articulo $=9188$

Saltos, Gladys., Pelegrín, Norberto. y Esquivel, Renier. (2017). La innovación tecnológica en la vinculación universidad-empresa-gobierno en el Ecuador y su influencia social. REFCalE: Revista Electrónica Formación y Calidad Educativa, 5(2). Recuperado de http://refcale.uleam.edu.ec/index.php/refcale/article/view/1794/978

Sanabria, Ana. y Hernández, Carmen. (2011). Percepción de los estudiantes y profesores sobre el uso de las TIC en los procesos de cambio e innovación en la enseñanza superior. Aloma: Revista de Psicologia, Ciències de l'educació i de l'esport, (29). Recuperado de http://www.revistaaloma.net/index.php/aloma/article/view/106

Sánchez, Luis., Reyes, Ana María., Ortiz, Diana., y Olarte, Fredy. (2018). El rol de la infraestructura tecnológica en relación con la brecha digital y la alfabetización digital en 100 instituciones educativas de Colombia. Calidad En La Educación, (47), 112-144. https://doi.org/10.31619/caledu.n47.32

Suárez, Ana., Pérez, Claudia., Vergara, María., y Alférez, Vìctor. (2015). Desarrollo de la lectoescritura mediante TIC y recursos educativos abiertos. Apertura: Revista de Innovación Educativa, $\quad 7(1)$ Recuperado de http://www.udgvirtual.udg.mx/apertura/index.php/apertura/article/view/595/412

Secretaría de Educación Superior, Ciencia, Tecnología e Innovación. (2019). Informe sobre la metodología de distribución de recursos destinados anualmente por parte del Estado a favor de las universidades y escuelas politécnicas públicas, públicas de posgrado y particulares que reciben rentas y asignaciones del Estado y su aplicación para el año 2020. Recuperado de https://www.epn.edu.ec/wp-content/uploads/2019/11/INFORMESENESCYT-CES-PRESUPUESTO-IES-2020.pdf 
Tamayo-Madrid, Tito. (2019). El sistema educativo de Ecuador: un sistema, dos mundos. Revista Andina de Educación 2(1), 8-17. Doi https://doi.org/10.32719/26312816.2019.2.1.2

Tapia, Hugo. (2018). Actitud hacia las TIC y hacia su integración didáctica en la formación inicial docente. Actualidades Investigativas en Educación, 18(3). Doi $\underline{10.15517 / a i e . v 18 \text { i3.34437 }}$

Velasteguí, Pablo. (2017). Plataformas virtuales y su impacto en la Educación Superior. Explorador Digital. Recuperado de http://cienciadigital.org/revistacienciadigital2/index.php/exploradordigital/article/downloa $\underline{\mathrm{d} / 318 / 730 /}$

Zambrano, Denny. y Zambrano, María. (2019). Las tecnologìas de la información y las comunicaciones (TICs) en la Educación Superior: Consideraciones teóricas. Revista Electrónica Formación y Calidad Educativa (REFCalE), 7(1), 213-228. Recuperado de file:///C:/Users/PC162/Downloads/2750-6597-1-PB.pdf 


\section{Anexo 1}

El cuestionario digital publicado en uvirtual@uce.edu.ec, al ser exportado a formato Word, se presenta de la siguiente manera:

\section{1}

¿Los docentes utilizan aparatos electrónicos para el proceso de enseñanza? MÉTODOS INNOVADORES d > > > >1/ SIEMPRE |2/ CASI SIEMPRE |3/ OCASIONALMENTE |4/ NUNCA $\mathrm{h}$

0 3925

¿La plataforma virtual institucional es utilizada en forma permanente para el desarrollo de las asignaturas?

MÉTODOS INNOVADORES

d >>>>>1/ SIEMPRE |2/ CASI SIEMPRE |3/ OCASIONALMENTE |4/ NUNCA

$\mathrm{h}$

0

3926

¿Los docentes crean blogs o medios similares para el desarrollo de las clases?

MÉTODOS INNOVADORES

d >>>>>1/ SIEMPRE |2/ CASI SIEMPRE |3/ OCASIONALMENTE |4/ NUNCA

$\mathrm{h}$

0

3934

¿Los docentes manifiestan predisposición para utilizar elementos tecnológicos en el proceso enseñanza aprendizaje?

METODOS INNOVADORES

d $>>>>>1 /$ SIEMPRE |2/ CASI SIEMPRE |3/ OCASIONALMENTE |4/ NUNCA

$\mathrm{h}$

0

3963

Los docentes utilizan diversas estrategias innovadoras para motivar su aprendizaje METODOS INNOVADORES

d >>>>>1/ SIEMPRE |2/ CASI SIEMPRE |3/ OCASIONALMENTE |4/ NUNCA

$\mathrm{h}$

0

3920

El desarrollo de asignaturas se lo realiza de manera tradicional, es decir, uso exclusivo del pizarrón

MÉTODOS INNOVADORES

d > > > > 1/ SIEMPRE |2/ CASI SIEMPRE |3/ OCASIONALMENTE |4/ NUNCA $\mathrm{h}$

0 


\section{3}

Considera usted que ¿es necesario la creación de redes sociales con fines académicos? MĖTODOS INNOVADORES d >>>>>1/ SIEMPRE |2/ CASI SIEMPRE |3/ OCASIONALMENTE |4/ NUNCA h 
Según su criterio ¿El uso de recursos tecnológicos provoca distracción en el proceso de aprendizaje?

METODO APRENDIZAJE

$d>>>>>1 /$ SIEMPRE |2/ CASI SIEMPRE |3/ OCASIONALMENTE |4/ NUNCA

h

0

3937

El uso diario de TIC en su proceso de aprendizaje, estaría alrededor de:

METODO APRENDIZAJE

d >>> > > menos de 1 hora |1-2 horas |2-4 horas $\mid 4$ o más horas

h

0

3938

El uso de tecnología apoya para la realización del trabajo autónomo (fuera del aula)

METODO DE APRENDIZAJE

d >>>>>1/ SIEMPRE |2/ CASI SIEMPRE |3/ OCASIONALMENTE |4/ NUNCA

$\mathrm{h}$

0

3918

¿El uso de TIC fomenta sus habilidades comunicativas?

HABILIDADES COMUNICATIVAS

d $>>>>>1 /$ SIEMPRE |2/ CASI SIEMPRE |3/ OCASIONALMENTE |4/ NUNCA

$\mathrm{h}$

0

3933

La utilización de dispositivos tecnológicos en el proceso de enseñanza, facilita una mejor comunicación con los docentes

HABILIDAD COMUNICATIVA

d > > > >1/ SIEMPRE |2/ CASI SIEMPRE |3/ OCASIONALMENTE |4/ NUNCA

h

0

3917

¿La inclusión de la tecnología en el proceso de aprendizaje, ha permitido mejoramiento en los procesos de investigación?

HABILIDADES INVESTIGACIÓN

d > > > >1/ SIEMPRE |2/ CASI SIEMPRE |3/ OCASIONALMENTE |4/ NUNCA

h

0

3921

La calidad de su aprendizaje se apoya en el uso de tecnologías de información y comunicación

HABILIDAD OPERATIVA

d >>>>>1/ SIEMPRE |2/ CASI SIEMPRE |3/ OCASIONALMENTE |4/ NUNCA

$\mathrm{h}$

0

Los contenidos de este artículo están bajo una licencia Creative Commons 


\section{6}

La tecnología le permite el desarrollo de autonomía en el aprendizaje HABILIDADES AUTONOMÍA d > > > > 1/ SIEMPRE |2/ CASI SIEMPRE |3/ OCASIONALMENTE |4/ NUNCA $\mathrm{h}$

0

3932

El uso de tecnología en el proceso de aprendizaje, desarrolla mejores relaciones entre el grupo de estudio? HABILIDADES INTERPERSONALES

d > > > > 1/ SIEMPRE |2/ CASI SIEMPRE |3/ OCASIONALMENTE |4/ NUNCA

$\mathrm{h}$

0

3935

Su dispositivo móvil, apoya para el desarrollo de su aprendizaje MÉTODOS DE APRENDIZAJE d >>>>>1/ SIEMPRE |2/ CASI SIEMPRE |3/ OCASIONALMENTE |4/ NUNCA $\mathrm{h}$

0

3936

El acceso a internet, es por: ACCESIBILIDAD d > >>> 1/PLAN DE DATOS |2/ INSTITUCIONAL |3/ HOGAR |4/ RECARGAS $\mathrm{h}$

0 
Revista indizada en
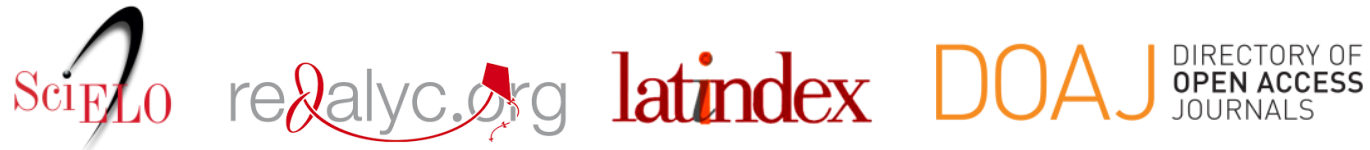

Distribuida en las bases de datos:

๑ Dialnet

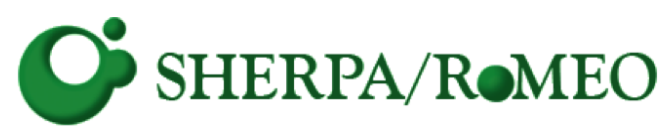

REDIB

Red Iberoamericana

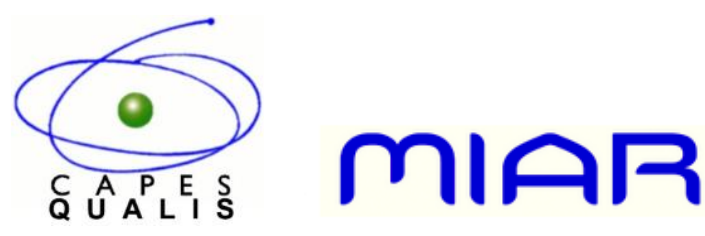

